\title{
Microstructural characterization of the Ti-30Nb-6Sn alloy synthesized by mechanical alloying
}

\author{
Elpidio Jiménez ${ }^{1}$, Luis Béjar ${ }^{2}$, Claudio Aguilar ${ }^{3}$, Ismeli Alfonso ${ }^{4}$ and Orlando Hernandez ${ }^{2}$ \\ ${ }^{1}$ Student, Morelia, Michoacan de Ocampo, Mexico, ${ }^{2}$ Professor, Morelia, Michoacan de Ocampo, Mexico, \\ ${ }^{3}$ Universidad Técnica Federico Santa Maria, Valparaíso, Valparaiso, Chile, ${ }^{4}$ Universidad Nacional \\ Autónoma de México, Morelia, Michoacan de Ocampo, Mexico
}

Titanium and its alloys are used as technical materials mainly because of the low density $(\rho=4.5 \mathrm{~g} \mathrm{~cm}-3)$ of Ti at technically useful levels of mechanical properties, and the formation of a passivating, protective oxide layer in air, which leads to a pronounced stability in corrosive media and at elevated temperatures.[1] The great interest of titanium alloys in the biomedical sector is well known due to their suitable characteristics for biomaterials compared to other metallic materials. However, there are still some concerns regarding titanium alloys such as the high Young's modulus which is superior to that of human bone, the design of $\beta$-Ti alloys emerges as a response to the necessity of improving other poorer aspects such as stiffness. $\beta$-Ti alloys have low Young's modulus while maintaining or enhancing the material strength by incorporating biocompatible elements such as $\mathrm{Nb}$ or Mo which makes them ideal for biomedical applications. Furthermore, Mo and $\mathrm{Nb}$ exhibit complete solubility in Ti above $882^{\circ} \mathrm{C}$ which allows a microstructural change and thus, the modification of their properties[2]. Mechanical alloying (MA) is a solid-state powder processing technique involving repeated welding, fracturing, and rewelding of powder particles in a high-energy ball mill. Originally developed to produce oxide-dispersion strengthened (ODS) nickel- and iron-base superalloys for applications in the aerospace industry, MA has now been shown to be capable of synthesizing a variety of equilibrium and non-equilibrium alloy phases starting from blended elemental or prealloyed powders. The non-equilibrium phases synthesized include supersaturated solid solutions, metastable crystalline and quasicrystalline phases, nanostructures, and amorphous alloys[3].

In this work, the microstructural characterization and chemical analysis are presented the Ti-30Nb-6Sn alloy was synthesized for $10 \mathrm{~h}$ by MA. The raw material used was: Titanium (Ti) as the main element, Niobium as a betagene element $(\mathrm{Nb})$, and Tin $(\mathrm{Sn})$ as a neutral element, this element reduces the elastic modulus with increasing percentages, its melting point is relatively low $\left(232^{\circ} \mathrm{C}\right)$ [4], and it has been proven that it diffuses well during sintering in addition to helping the diffusion of niobium in sintering stage[5]. In the X-ray study, the crystalline structures of the elements and the microstructural changes in the mechanical alloying were identified through diffractograms. The aliquots were analyzed in an X-ray diffractometer, Brand: STOE, Model: STADI-MP, Max kV: $40 \mathrm{~mA}$ Max: 30, Radiation $1.54060 \lambda(\AA) \mathrm{Cu}$. Reading conditions: By Transmission, 2 Theta with angles from $10^{\circ}$ to $100^{\circ}$, measurement time 15 min., Step $=2.1$. The morphology was analyzed by means of scanning electron microscopes (SEM), while the chemical composition was evaluated by means of energy dispersion spectroscopy (EDS).

Figure 1 shows the X-Ray results of the alloy. It is possible to observe the presence of different peaks, which correspond to the $\beta-\mathrm{Ti}, \mathrm{Nb}$, and $\mathrm{Sn}$ elements. The Match program was used to determine the phases of the elements

Figure 2a shows the morphology of the particles analyzed by SEM. It can be observed a different morphology of the particles; the average size is ranking $10 \mu \mathrm{m}$. The chemical composition of the particles can be observed in figure $2 b$ where the stoichiometric distribution of the elements is seen, the homogeneous distribution of titanium, and the agglomerations of niobium and tin are observed, in addition to the presence of the elements that made up the grinding medium. 


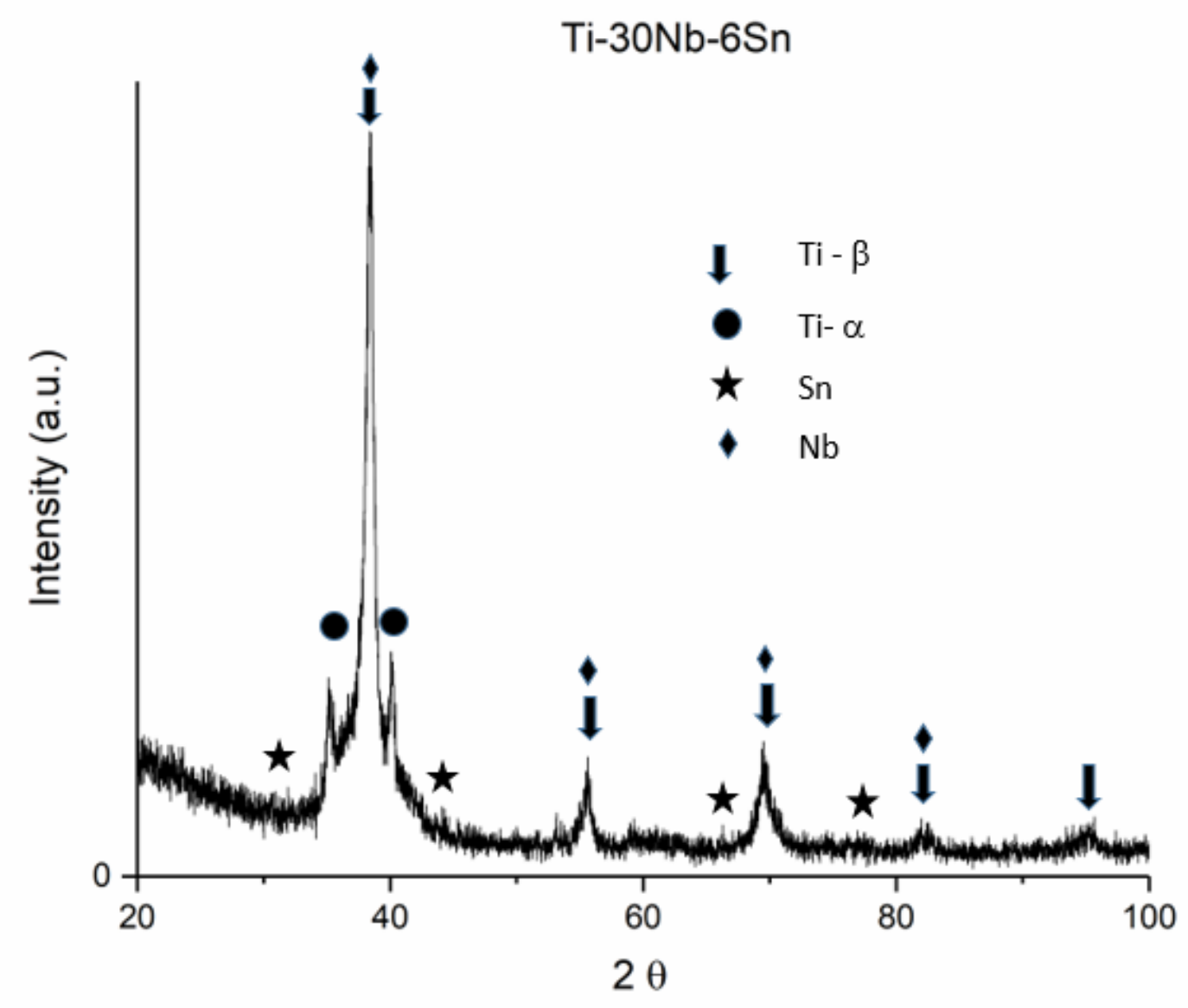

Figure 1. Figure 1. XRD analysis of Ti-30Nb-6Sn \%at alloy milled for $10 \mathrm{~h}$

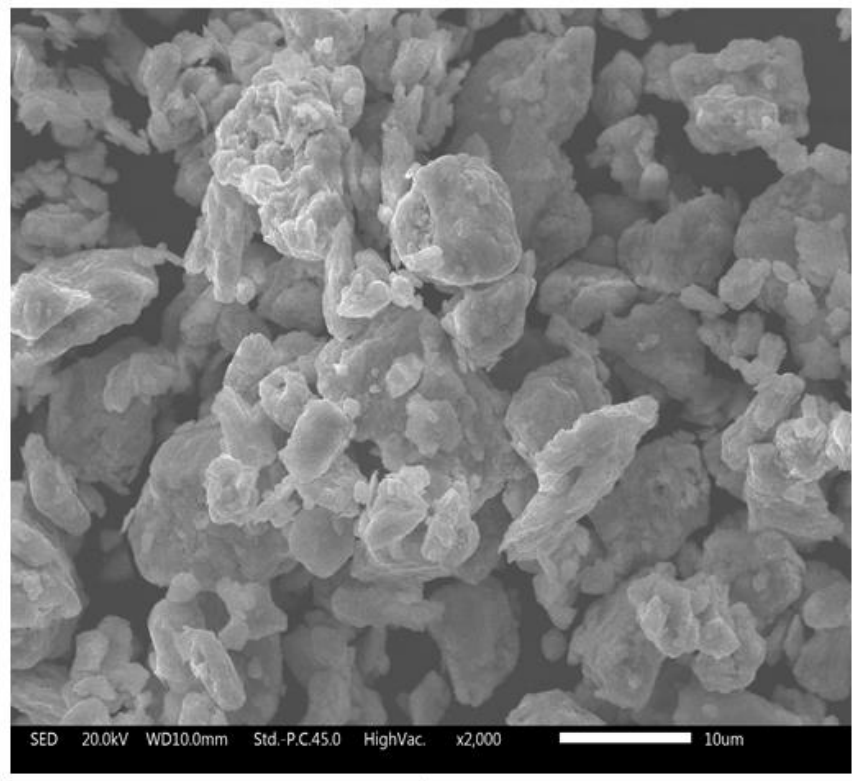

a)

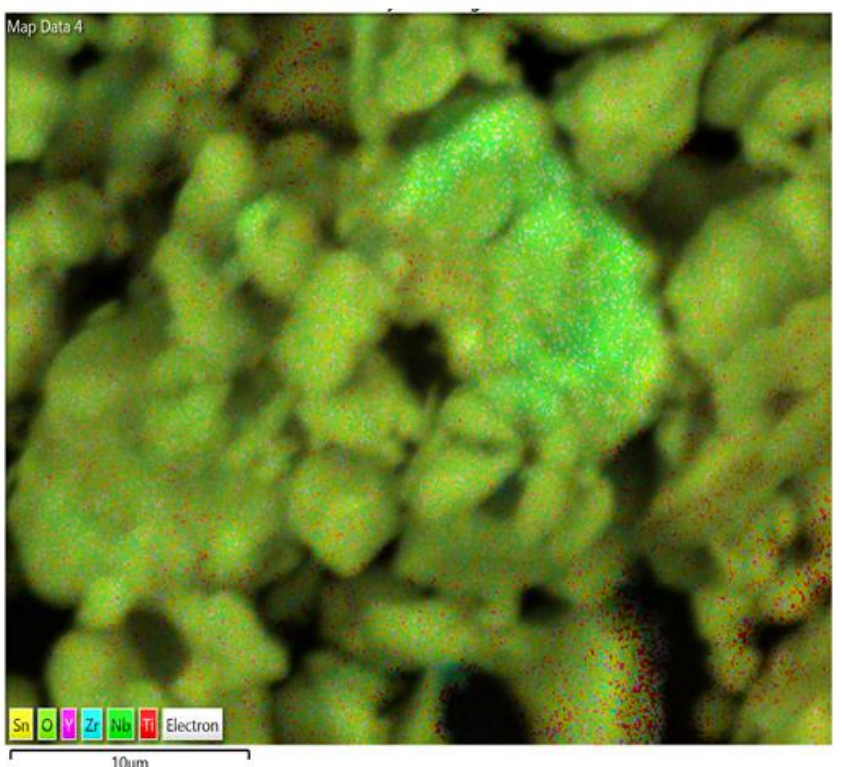

b)

Figure 2. Figure 2. Ti-30Nb-6Sn alloy milled for $10 \mathrm{~h}$ a) SEM image and b) EDS 


\section{References}

[1] H. Warlimont and W. Martienssen, "Titanium and Titanium alloys." 2018.

[2] J. Ureña, et al., "Role of beta-stabilizing elements on the microstructure and mechanical properties evolution of modified PM Ti surfaces designed for biomedical applications," 2018.

[3] C. Suryanarayana, "Mechanical alloying and milling," 2004.

[4] S. Hanada, et al., "Mechanical compatibility of titanium implants in hard tissues,", 2005.

[5] X. Wang, et al, "Ti-Nb-Sn-hydroxyapatite composites synthesized by mechanical alloying and high frequency induction heated sintering," 2011. 